\title{
Total Return Swap Valuation with Counterparty Risk and Interest Rate Risk
}

\author{
Anjiao Wang ${ }^{1}$ and Zhongxing Ye $e^{1,2}$ \\ ${ }^{1}$ School of Business Information, Shanghai University of International Business and Economics, Shanghai 201620, China \\ ${ }^{2}$ Department of Mathematics, Shanghai Jiaotong University, Shanghai 200240, China
}

Correspondence should be addressed to Anjiao Wang; anjiaowang@126.com

Received 17 January 2014; Accepted 28 April 2014; Published 28 May 2014

Academic Editor: Imran Naeem

Copyright (c) 2014 A. Wang and Z. Ye. This is an open access article distributed under the Creative Commons Attribution License, which permits unrestricted use, distribution, and reproduction in any medium, provided the original work is properly cited.

\begin{abstract}
We study the pricing of total return swap (TRS) under the contagion models with counterparty risk and the interest rate risk. We assume that interest rate follows Heath-Jarrow-Morton (HJM) forward interest rate model and obtain the Libor market interest rate. The cases where default is related to the interest rate and independent of interest rate are considered. Using the methods of change of measure and the "total hazard construction," the joint default probabilities are obtained. Furthermore, we obtain the closed-form formulas of TRS under different contagion models, respectively.
\end{abstract}

\section{Introduction}

Total return swap (TRS), as a type of credit derivatives and a financing and leverage tool, is an important offbalance sheet tool, particularly for hedge funds and for banks seeking additional fee income. The corporate bonds and their credit derivatives are typically financial tools in the markets which undertake and avoid the credit risk of the companies. Therefore, the key of management of credit risk is the fair pricing of credit derivatives. Especially after 2007 financing crisis, the contagion effect of credit risk has attracted huge attention of financial market regulators and financial institutions. To price credit derivatives, default contagion models are developed rapidly in order to make them more realistic after the subprime mortgage crisis.

The approaches of modeling the pricing of credit derivatives are mainly the value-of-the-firm (or structural) approach and the intensity-based approach (or reduced form approach). The structural model is based on the work of Merton [1], Black and Cox [2], and Geske [3]: the default occurs when the firm assets are insufficient to meet payments on debt or the value of the firm asset falls below a prespecified level.

Reduced-form models are developed by Artzner and Delbaen [4], Duffie et al. [5], Jarrow and Turnbull [6], and
Madan and Unal [7]. Duffie and Lando [8] show that a reduced-form model can be obtained from a structural model with incomplete accounting information. The simplest type of reduced-form model is where the default time or the credit migration is the first jump of an exogenously given jump process with an intensity. In Jarrow et al. [9], the intensity for credit migration is constant; see also Litterman and Iben [10] for a Markov chain model of credit migration. In the papers by Duffie et al. [5], Duffie and Singleton [11], and Lando [12], the intensity of default is a random process. The common feature of the reduced-form models is that default cannot be predicted and can occur at any time. Therefore, reduced-form models have been used to price a wide variety of instruments. In recent years, some papers on estimating the parameters of these models are given by Collin-Dufresne and Solnik [13] and Duffee [14]. Jarrow and Yu [15] set up a reduced-form model in which estimation can be based on bond prices as well as credit default swap prices. A systematic development of mathematical tools for reduced-form models has been given by Elliott et al. [16]. Jamshidian [17] develops change of numeraire methodology for reduced-form models.

A TRS is a bilateral financial contract between a total return payer and a total return receiver. One party (the total return payer) pays the total return of a reference security (or reference securities) and receives a form of payment from 


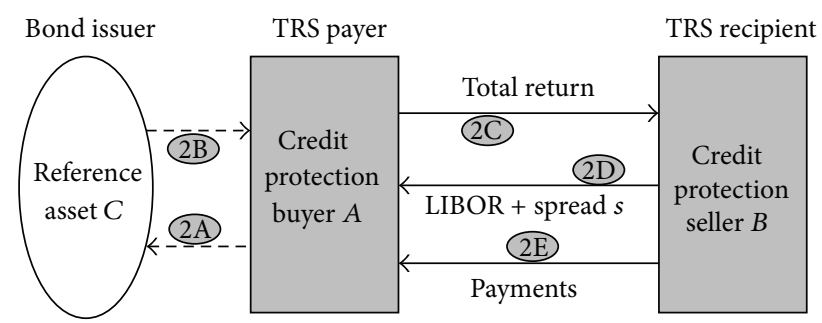

FIgURE 1: The structure of TRS.

the other party (the receiver of the total rate of return). Often payment is a floating rate payment, a spread to LIBOR. The reference assets can be indices, bonds (emerging market, sovereign, bank debt, mortgage-backed securities, or corporate), loans (term or revolver), equities, real estate receivables, lease receivables, or commodities. Ye and Zhuang [18] consider the pricing of TRS only when reference asset defaults; they obtain TRS pricing formula when default is independent of the interest rate. When default is related to the interest rate, using the hybrid model given in Das and Sundaram [19], they model default time and the interest rate and give the Monte Carlo simulation result.

In this paper, we consider the counterparty default contagion risk between total return receiver (firm $B$ ) and the reference asset (firm $C$ ) and study two-firm contagion models. The cash flow of a TRS in this model is provided by Figure 1.

Suppose that firm $A$ (a corporate bond investing firm, credit protection buyer) holds a corporate bond (reference asset) issued by firm $C$ (a corporate bond issuer) (refer to $2 \mathrm{~A}$ in Figure 1), and firm $C$ is subject to default. At bond maturity, if firm $C$ does not default, it will pay the bond principle and interest to firm $A$ (refer to $2 \mathrm{~B}$ ). On the other hand, to hedge the default risk of firm $C$, firm $A$ and firm $B$ (credit protection seller, subject to default also) enter into a TRS contract. If firm $C$ has no default, firm $A$ will make its total return to firm $B$ (refer to 2C), and, in exchange, firm $B$ gives the Libor plus a spread $s$ to $A$ (refer to $2 \mathrm{D}$ ). Firm $B$ promises to compensate $A$ for its loss in the event of default of firm $C$ (refer to $2 \mathrm{E}$ ).

The structure of this paper is organized as follows. In Section 2, we give the basic setup and HJM forward interest rate model. In Section 3, we study the two-firm contagion models when default is independent of stochastic interest rate and obtain the closed-form formulas of TRS. In Section 4, we consider the case that default is related to interest rate. Using the "total hazard" approach, joint survival probabilities are derived and analytic formulas are obtained under two-firm contagion models. Section 5 is the conclusion.

\section{Basic Setup and HJM Forward Interest Rate Model}

We consider a filtered probability space $\left(\Omega, \mathscr{F},\left\{\mathscr{F}_{t}\right\}_{t=0}^{T^{*}}, P\right)$ which is an uncertain economy with a time horizon of $T^{*}$, satisfying the usual conditions of right-continuity and completeness with respect to $P$-null sets, where $\mathscr{F}=\mathscr{F}_{T^{*}}$ and $P$ is an equivalent martingale measure under which discounted bond prices are martingales. We assume the existence and uniqueness of $P$ so that bond markets are complete and there is no arbitrage, as shown in discrete time case by Harrison and Kreps [20] and in continuous time case by Harrison and Pliska [21]. Subsequent specifications of the model are all under the equivalent martingale measure (or risk neutral measure) $P$.

On this probability space there is an $\mathbb{R}^{d}$-valued process $X_{t}$, which presents $d$ dimensional economy-wide state variables. In this paper, we consider the only one state variable which is the interest rate denoted by $r_{t}$. There are also two point processes, $N^{i}(i=B, C)$, initialized at 0 , representing the default processes of the firm $B$ and firm $C$, respectively, such that the default of the firm $i$ occurs when $N^{i}$ jumps from 0 to 1 .

According to the information contained in the state variables and the default processes, the enlarged filtration is defined by

$$
\mathscr{F}_{t}=\mathscr{F}_{t}^{r} \vee \mathscr{F}_{t}^{B} \vee \mathscr{F}_{t}^{C},
$$

where

$$
\begin{gathered}
\mathscr{F}_{t}^{r}=\sigma\left(r_{s}, 0 \leq s \leq t\right), \\
\mathscr{F}_{t}^{i}=\sigma\left(N_{s}^{i}, 0 \leq s \leq t\right), \quad i=B, C,
\end{gathered}
$$

are the filtrations generated by $r_{t}$ and $N_{t}^{i}(i=B, C)$, respectively.

Let

$$
\begin{aligned}
& \mathscr{G}_{t}^{B}=\mathscr{F}_{t}^{B} \vee \mathscr{F}_{T^{*}}^{r} \vee \mathscr{F}_{T^{*}}^{C}=\mathscr{F}_{t}^{B} \vee \mathscr{G}_{0}^{-B}, \\
& \mathscr{G}_{t}^{C}=\mathscr{F}_{t}^{C} \vee \mathscr{F}_{T^{*}}^{r} \vee \mathscr{F}_{T^{*}}^{B}=\mathscr{F}_{t}^{C} \vee \mathscr{G}_{0}^{-C},
\end{aligned}
$$

where

$$
\mathscr{G}_{0}^{-B}=\mathscr{F}_{T^{*}}^{r} \vee \mathscr{F}_{T^{*}}^{C}, \quad \mathscr{G}_{0}^{-C}=\mathscr{F}_{T^{*}}^{r} \vee \mathscr{F}_{T^{*}}^{B} .
$$

$\mathscr{G}_{0}^{-i}(i=B, C)$ contains complete information on the state variables and the default processes of all firms up to time $T^{*}$ other than that of the $i$ th firm.

Let $\tau^{i}$ denote the default time of firm $i$; namely, $\tau^{i}$ is the first jump time of $N^{i}$, which can be defined as

$$
\tau^{i}=\inf \left\{t: \int_{0}^{t} \lambda_{s}^{i} d s \geq E^{i}\right\}
$$

where $\left\{E^{i}\right\}(i=B, C)$ is independent of $r_{t}\left(t \in\left[0, T^{*}\right]\right)$.

According to the Doob-Meyer decomposition, we have that

$$
M_{t}^{i}=N_{t}-\int_{0}^{t \wedge \tau^{i}} \lambda_{s}^{i} d s
$$

is a $\left(P, \mathscr{F}_{t}\right)$-martingale.

Due to the impact of counterparty risk, the default intensities $\lambda_{t}^{B}$ and $\lambda_{t}^{C}$ of firms $B$ and $C$ are no longer independent under the condition $\mathscr{F}_{t}^{r}$. The conditional survival probability 
and the unconditional survival probability of firm $i$ are given by

$$
\begin{gathered}
P\left(\tau^{i}>t \mid \mathscr{G}_{0}^{-i}\right)=\exp \left(-\int_{0}^{t} \lambda_{s}^{j} d s\right), \quad t \in[0, T], \\
P\left(\tau^{i}>t\right)=E\left[\exp \left(-\int_{0}^{t} \lambda_{s}^{i} d s\right)\right], \quad t \in[0, T],
\end{gathered}
$$

respectively.

As a type of market risk, interest rate risk analysis is almost always based on simulating movements in one or more yield curves using the Heath-Jarrow-Morton (HJM) framework to ensure that the yield curve movements are both consistent with current market yield curves and such that no riskless arbitrage is possible. HJM model was developed in early 1992 by Heath et al. [22]. In this paper, we assume the default free interest rate is stochastic forward interest rate $f(t, T)$, which follows the HJM model

$$
d f(t, T)=\alpha(t, T) d t+\sigma(t, T) d W_{t}, \quad 0 \leq t \leq T,
$$

where $\alpha(t, T)$ is the drift term and $\sigma(t, T)$ is the volatility term, and they are both stochastic. For every fixed time $T$, they are adapted processes to $\mathscr{F}_{t}$.

The arbitrage free condition [23] shows that the Brown movement under each real probability measure can be transformed into the Brown movement under the risk neutral measure. For simplification, in this paper, we assume the Brown movement $W_{t}$ is standard under the risk neutral measure $P$, where $\alpha(t, T)$ satisfies

$$
\alpha(t, T)=\sigma(t, T) \sigma^{*}(t, T)=\sigma(t, T) \int_{t}^{T} \sigma(t, v) d v .
$$

The solution of (9) is

$$
f(t, T)=f(0, T)+\int_{0}^{t} \alpha(u, T) d u+\int_{0}^{t} \sigma(u, T) d W_{u},
$$

where $f(0, T)$ is the initial forward interest rate curve, which is known.

At time $t$ the instantaneous interest rate $r_{t}=f(t, t)$. $D(t)=\exp \left(-\int_{0}^{t} r_{u} d u\right)$ is the discounted process. Let $B(t, T)$ be the time- $t$ value of a zero-coupon bond with face value 1 at maturity $T$; we have

$$
B(t, T)=\exp \left(-\int_{t}^{T} f(t, v) d v\right), \quad 0 \leq t \leq T \leq \bar{T} .
$$

Under the risk neutral measure $P, B(t, T)$ satisfies the following differential equation:

$$
d B(t, T)=r_{t} B(t, T) d t-\sigma^{*}(t, T) B(t, T) d W_{t} .
$$

Denote $L(t, T)$ to be the time- $t$ locked investment yield curve from $T$ to $T+\delta$; then,

$$
L(t, T)=\frac{B(t, T)-B(t, T+\delta)}{\delta B(t, T+\delta)} .
$$

For $0 \leq t<T, L(t, T)$ is called forward Libor interest rate, and, for $t=T, L(t, T)$ is called spot Libor interest rate. $\delta$ is the duration of Libor, normally 0.25 year or half year.

In the next two sections of this paper, we discuss the pricing of TRS with default risk and interest rate risk under different contagion models.

\section{TRS Valuation When Default Is Independent of the Interest Rate}

Assume that reference asset $C$ is a defaultable coupon bond with face value 1 and the same maturity $T$ with TRS contract. $T_{0}, T_{1}, T_{2}, \ldots, T_{n}$ are bond interest payment dates, where $0=$ $T_{0}<T_{1}<\cdots<T_{n}=T$, for $0 \leq i \leq n-1, T_{i+1}-T_{i}=\Delta T$, $n \Delta T=T$.

Denote $C_{i}$ to be the time- $T_{i}$ cash flow of TRS payer, where $C_{1}, \ldots, C_{n-1}$ are the interest payments at time $T_{i}(i=$ $1, \ldots, n-1)$ and $C_{n}$ is the sum of interest payment and valueadded of the bond at time $T_{n}=T$, which are all determined at $T_{0}=0$. Let $M$ be notional principal, and $\delta$ is the maturity of Libor interest rate, the same with bond's payment cycle $\Delta T$, namely, $\delta=\Delta T$. For simplification, the default recovery rate of reference asset $C$ is 0 .

At time 0 , for TRS payment leg, the discounted expectation of the cash flow $F_{B}$ is

$$
E\left[F_{B}\right]=E\left[\sum_{i=1}^{n} D\left(T_{i}\right) C_{i} 1_{\left\{\tau^{B} \wedge \tau^{\complement}>T_{i}\right\}}\right] .
$$

At time 0 , for TRS recipient leg, the discounted expectation of the cash flow $F_{C}$ is

$$
\begin{aligned}
E\left[F_{C}\right]= & E\left[\sum_{i=1}^{n} D\left(T_{i}\right) \delta M\left(L\left(T_{i-1}, T_{i-1}\right)+s\right) 1_{\left\{\tau^{B} \wedge \tau^{C}>T_{i}\right\}}\right] \\
& +E\left[D\left(\tau^{C}+\theta\right) 1_{\left\{\tau^{C} \leq T\right\}} 1_{\left\{\tau^{B}>\tau^{C}+\theta\right\}}\right],
\end{aligned}
$$

where $E$ is the expectation under risk neutral measure $P, \theta$ is the length of settlement period, and $\tau^{C}+\theta$ is settlement date. $L\left(T_{i-1}, T_{i-1}\right)$ is time- $T_{i-1}$ locked $T_{i}$-Libor interest rate. $s$ is the TRS spread and $D(\cdot)$ is the discounted factor.

We assume the market is complete; namely, there is no arbitrage. Therefore, according to the arbitrage-free principle, we have

$$
E\left[F_{B}\right]=E\left[F_{C}\right] .
$$


The general pricing formula of TRS is derived as

$$
\begin{aligned}
s=(E & {\left[\sum_{i=1}^{n} D\left(T_{i}\right) C_{i} 1_{\left\{\tau^{B} \wedge \tau^{C}>T_{i}\right\}}\right] } \\
& \left.-E\left[\sum_{i=1}^{n} D\left(T_{i}\right) \delta M L\left(T_{i-1}, T_{i-1}\right) 1_{\left\{\tau^{B} \wedge \tau^{C}>T_{i}\right\}}\right]\right) \\
& \times\left(E\left[\sum_{i=1}^{n} D\left(T_{i}\right) \delta M 1_{\left\{\tau^{B} \wedge \tau^{C}>T_{i}\right\}}\right]\right)^{-1} \\
- & \frac{E\left[D\left(\tau^{C}+\theta\right) 1_{\left\{\tau^{C} \leq T\right\}} 1_{\left\{\tau^{B}>\tau^{C}+\theta\right\}}\right]}{E\left[\sum_{i=1}^{n} D\left(T_{i}\right) \delta M 1_{\left\{\tau^{B} \wedge \tau^{C}>T_{i}\right\}}\right]} .
\end{aligned}
$$

3.1. TRS Valuation under Two-Firm Looping Default Contagion Model. In this subsection we assume that the default intensities of credit protection seller $B$ and reference asset $C$ are given, respectively, by

$$
\begin{aligned}
& \lambda_{t}^{B}=b_{0}+b_{1} 1_{\left\{\tau^{C} \leq t\right\}}, \\
& \lambda_{t}^{C}=c_{0}+c_{1} 1_{\left\{\tau^{B} \leq t\right\}},
\end{aligned}
$$

where $b_{0}$ and $b_{0}$ are nonnegative, satisfying $b_{0}+b_{1}>0$ and $c_{0}+c_{1}>0$.

With the result in Leung and Kwok [24], the joint survival probability and the joint density of default time $\left(\tau^{B}, \tau^{C}\right)$ are given by

$$
\begin{aligned}
& P\left(\tau^{B}>t_{1}, \tau^{C}>t_{2}\right) \\
& \quad= \begin{cases}e^{-b_{0} t_{1}-c_{0} t_{2}}\left[\frac{c_{0} e^{-b_{1}\left(t_{1}-t_{2}\right)}-b_{1} e^{-c_{0}\left(t_{1}-t_{2}\right)}}{c_{0}-b_{1}}\right], & t_{2} \leq t_{1} \\
e^{-b_{0} t_{1}-c_{0} t_{2}}\left[\frac{c_{0} e^{-c_{1}\left(t_{2}-t_{1}\right)}-c_{1} e^{-b_{0}\left(t_{2}-t_{1}\right)}}{b_{0}-c_{1}}\right], & t_{2}>t_{1},\end{cases}
\end{aligned}
$$

$$
\begin{aligned}
& f\left(t_{1}, t_{2}\right) \\
& \quad= \begin{cases}f_{1}\left(t_{1}, t_{2}\right)=c_{0}\left(b_{0}+b_{1}\right) e^{-\left(b_{0}+b_{1}\right) t_{1}-\left(c_{0}-b_{1}\right) t_{2}}, & t_{2} \leq t_{1} \\
f_{2}\left(t_{1}, t_{2}\right)=b_{0}\left(c_{0}+c_{1}\right) e^{-\left(c_{0}+c_{1}\right) t_{2}-\left(b_{0}-c_{1}\right) t_{1}}, & t_{2}>t_{1},\end{cases}
\end{aligned}
$$

respectively.

When the default process and the interest rate process are independent, by computing formula (18), we can obtain the following result.

Theorem 1. With the default intensity process (19)-(20) and HJM interest rate model (9), the price sof TRS is given by

$$
\begin{aligned}
s= & \frac{\sum_{i=1}^{n}\left(C_{i}-\delta M L\left(T_{i-1}, T_{i-1}\right)\right) B\left(0, T_{i}\right) e^{-\left(b_{0}+c_{0}\right) T_{i}}}{\sum_{i=1}^{n} \delta M e^{-\left(b_{0}+c_{0}\right) T_{i}}} \\
& -\frac{\mathcal{c}_{0} e^{-\left(b_{0}+b_{1}\right) \theta} \int_{0}^{T} B(0, v+\theta) e^{-\left(b_{0}+c_{0}\right) v} d v}{\sum_{i=1}^{n} \delta M e^{-\left(b_{0}+c_{0}\right) T_{i}}} .
\end{aligned}
$$

Proof. By the arbitrage free principle, the price formula (18) becomes

$$
\begin{aligned}
s=\left(E\left[\sum_{i=1}^{n} D\left(T_{i}\right) C_{i} 1_{\left\{\tau^{B} \wedge \tau^{C}>T_{i}\right\}}\right]\right. \\
\left.\quad-E\left[\sum_{i=1}^{n} D\left(T_{i}\right) \delta M L\left(T_{i-1}, T_{i-1}\right) 1_{\left\{\tau^{B} \wedge \tau^{C}>T_{i}\right\}}\right]\right) \\
\times\left(E\left[\sum_{i=1}^{n} D\left(T_{i}\right) \delta M 1_{\left\{\tau^{B} \wedge \tau^{C}>T_{i}\right\}}\right]\right)^{-1} \\
-\frac{E\left[D\left(\tau^{C}+\theta\right) 1_{\left\{\tau^{C} \leq T\right\}} 1_{\left\{\tau^{B}>\tau^{C}+\theta\right\}}\right]}{E\left[\sum_{i=1}^{n} D\left(T_{i}\right) M 1_{\left\{\tau^{B} \wedge \tau^{C}>T_{i}\right\}}\right]} .
\end{aligned}
$$

To obtain the analytic solution of $s$, we need to compute the following three expectation values:

$$
\begin{aligned}
K_{1} & :=\sum_{i=1}^{n} E\left[D\left(T_{i}\right) 1_{\left\{\tau^{B} \wedge \tau^{C}>T_{i}\right\}}\right], \\
K_{2} & :=\sum_{i=1}^{n} E\left[D\left(T_{i}\right) L\left(T_{i-1}, T_{i-1}\right) 1_{\left\{\tau^{B} \wedge \tau^{C}>T_{i}\right\}}\right], \\
K_{3} & :=E\left[D\left(\tau^{C}+\theta\right) 1_{\left\{\tau^{C} \leq T\right\}} 1_{\left\{\tau^{B}>\tau^{C}+\theta\right\}}\right],
\end{aligned}
$$

where

$$
\begin{aligned}
& K_{1}=\sum_{i=1}^{n} E\left[D\left(T_{i}\right) 1_{\left\{\tau^{B} \wedge \tau^{C}>T_{i}\right\}}\right] \\
& =\sum_{i=1}^{n} E\left[\exp \left(-\int_{0}^{T_{i}} r_{u} d u\right)\right] E\left[1_{\left\{\tau^{B} \wedge \tau^{C}>T_{i}\right\}}\right] \\
& =\sum_{i=1}^{n} \exp \left(-\int_{0}^{T_{i}} f(0, u) d u\right) E\left[1_{\left\{\tau^{B} \wedge \tau^{C}>T_{i}\right\}}\right] \\
& =\sum_{i=1}^{n} B\left(0, T_{i}\right) e^{-\left(b_{0}+c_{0}\right) T_{i}}, \\
& K_{2}=\sum_{i=1}^{n} E\left[D\left(T_{i}\right) L\left(T_{i-1}, T_{i-1}\right) 1_{\left\{\tau^{B} \wedge \tau^{C}>T_{i}\right\}}\right] \\
& =\sum_{i=1}^{n} B\left(0, T_{i}\right) L\left(T_{i-1}, T_{i-1}\right) e^{-\left(b_{0}+c_{0}\right) T_{i}}, \\
& K_{3}=E\left[D\left(\tau^{C}+\theta\right) 1_{\left\{\tau^{C} \leq T\right\}} 1_{\left\{\tau^{B}>\tau^{C}+\theta\right\}}\right] \\
& =E\left[E\left[D\left(\tau^{C}+\theta\right) 1_{\left\{\tau^{C} \leq T\right\}} 1_{\left\{\tau^{B}>\tau^{C}+\theta\right\}} \mid \mathscr{F}_{T^{*}}^{r}\right]\right] \\
& =E\left[B\left(0, \tau^{C}+\theta\right) 1_{\left\{\tau^{C} \leq T\right\}} 1_{\left\{\tau^{B}>\tau^{C}+\theta\right\}}\right]
\end{aligned}
$$




$$
\begin{aligned}
& =\int_{0}^{T} \int_{v+\theta}^{\infty} B(0, v+\theta) f_{2}(u, v) d u d v \\
& =\int_{0}^{T} B(0, v+\theta) \int_{v+\theta}^{\infty} c_{0}\left(b_{0}+b_{1}\right) e^{-\left(b_{0}+b_{1}\right) t_{1}-\left(c_{0}-b_{1}\right) t_{2}} d u d v \\
& =c_{0} e^{-\left(b_{0}+b_{1}\right) \theta} \int_{0}^{T} B(0, v+\theta) e^{-\left(b_{0}+c_{0}\right) v} d v .
\end{aligned}
$$

Substitute $K_{1}, K_{2}$, and $K_{3}$ into formula (24), and we obtain

$$
\begin{gathered}
s=\frac{\sum_{i=1}^{n}\left(C_{i}-\delta M L\left(T_{i-1}, T_{i-1}\right)\right) B\left(0, T_{i}\right) e^{-\left(b_{0}+c_{0}\right) T_{i}}}{\sum_{i=1}^{n} \delta M e^{-\left(b_{0}+c_{0}\right) T_{i}}} \\
-\frac{c_{0} e^{-\left(b_{0}+b_{1}\right) \theta} \int_{0}^{T} B(0, v+\theta) e^{-\left(b_{0}+c_{0}\right) v} d v}{\sum_{i=1}^{n} \delta M e^{-\left(b_{0}+c_{0}\right) T_{i}}} .
\end{gathered}
$$

We complete the proof.

Remark 2. From (23), we can conclude that when the length $\theta$ of settlement period is zero, the swap rate $s$ is only related to the systematic factors $b_{0}$ and $c_{0}$, irrelative to the default contagion between two firms.

\subsection{TRS Valuation under Two-Firm Attenuation Contagion} Model. In this subsection, we consider the default contagion to have the hyperbolic attenuation effect. Default intensities of firms $B$ and $C$ have the following forms:

$$
\begin{aligned}
& \lambda_{t}^{B}=b_{0}+\frac{b_{2}}{b_{1}\left(t-\tau^{C}\right)+1} 1_{\left\{\tau^{C} \leq t\right\}}, \\
& \lambda_{t}^{C}=c_{0}+\frac{c_{2}}{c_{1}\left(t-\tau^{B}\right)+1} 1_{\left\{\tau^{B} \leq t\right\}},
\end{aligned}
$$

where $b_{0}, c_{0}, b_{1}$, and $c_{1}$ are nonnegative, satisfying $b_{0}+b_{2}>0$ and $c_{0}+c_{2}>0 . b_{2}$ and $c_{2}$ reflect the impact strength of the counterparty and $b_{1}$ and $c_{1}$ show the attenuation speed of one party default on the other party. For $b_{1}=c_{1}=0$, the model becomes the looping default model (19)-(20).

Under the above model, the analytic solutions of the joint survival probability cannot be obtained. For simplification, we assume $b_{2}=-b_{1}$; then, (28) and (29) become, respectively,

$$
\begin{aligned}
& \lambda_{t}^{B}=b_{0}-\frac{b_{1}}{b_{1}\left(t-\tau^{C}\right)+1} 1_{\left\{\tau^{C} \leq t\right\}}, \\
& \lambda_{t}^{C}=c_{0}-\frac{c_{1}}{c_{1}\left(t-\tau^{B}\right)+1} 1_{\left\{\tau^{B} \leq t\right\}} .
\end{aligned}
$$

According to the result in Bai et al. [25], with the intensities (30)-(31), the joint survival probability and the joint density function of $\left(\tau^{B}, \tau^{C}\right)$ are given by

$$
\begin{aligned}
& P\left(\tau^{B}>t_{1}, \tau^{C}>t_{2}\right)
\end{aligned}
$$

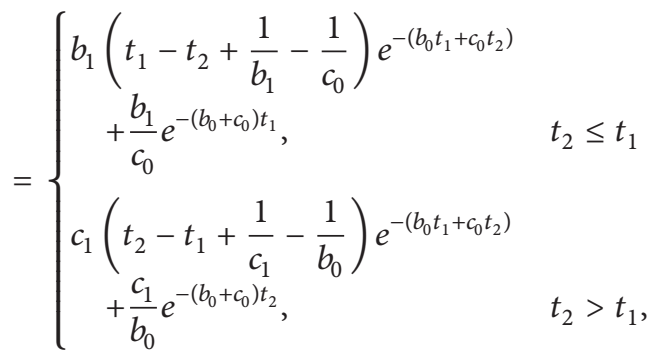

$$
\begin{aligned}
& f\left(t_{1}, t_{2}\right) \\
& =\left\{\begin{array}{ccc}
f_{1}\left(t_{1}, t_{2}\right)=c_{0} b_{0} b_{1}\left[t_{1}-t_{2}+\frac{1}{b_{1}}-\frac{1}{b_{0}}\right] & \\
& \times e^{-\left(b_{0} t_{1}+c_{0} t_{2}\right)}, & t_{2} \leq t_{1} \\
f_{2}\left(t_{1}, t_{2}\right)=b_{0} c_{0} c_{1}\left[t_{2}-t_{1}+\frac{1}{c_{1}}-\frac{1}{c_{0}}\right] & \\
& \times e^{-\left(b_{0} t_{1}+c_{0} t_{2}\right)}, & t_{2}>t_{1},
\end{array}\right.
\end{aligned}
$$

respectively.

Then the expression of $s$ is given by the following theorem.

Theorem 3. With the default intensities (30)-(31) and HJM interest rate model (9), $s$ is given by

$$
\begin{aligned}
s= & \frac{\sum_{i=1}^{n} C_{i} B\left(0, T_{i}\right) e^{-\left(b_{0}+c_{0}\right) T_{i}}}{\sum_{i=1}^{n} \delta M B\left(0, T_{i}\right) e^{-\left(b_{0}+c_{0}\right) T_{i}}} \\
& -\frac{\sum_{i=1}^{n} L\left(T_{i-1}, T_{i-1}\right) \delta M B\left(0, T_{i}\right) e^{-\left(b_{0}+c_{0}\right) T_{i}}}{\sum_{i=1}^{n} \delta M B\left(0, T_{i}\right) e^{-\left(b_{0}+c_{0}\right) T_{i}}} \\
& -\frac{c_{0}\left(1+b_{1} \theta\right) e^{-b_{0} \theta} \int_{0}^{T} B(0, v+\theta) e^{-\left(b_{0}+c_{0}\right) v} d v}{\sum_{i=1}^{n} \delta M B\left(0, T_{i}\right) e^{-\left(b_{0}+c_{0}\right) T_{i}}} .
\end{aligned}
$$

Proof. To compute (18), we need to only compute the following three expectation values:

$$
\begin{aligned}
& \widetilde{K}_{1}:=\sum_{i=1}^{n} E\left[D\left(T_{i}\right) 1_{\left\{\tau^{B} \wedge \tau^{C}>T_{i}\right\}}\right], \\
& \widetilde{K}_{2}:=\sum_{i=1}^{n} E\left[D\left(T_{i}\right) L\left(T_{i-1}, T_{i-1}\right) 1_{\left\{\tau^{B} \wedge \tau^{C}>T_{i}\right\}}\right], \\
& \widetilde{K}_{3}:=E\left[D\left(\tau^{C}+\delta\right) 1_{\left\{\tau^{C} \leq T\right\}} 1_{\left\{\tau^{B}>\tau^{C}+\delta\right\}}\right],
\end{aligned}
$$


where

$$
\begin{aligned}
\widetilde{K}_{1}= & \sum_{i=1}^{n} E\left[D\left(T_{i}\right) 1_{\left\{\tau^{B} \wedge \tau^{C}>T_{i}\right\}}\right] \\
= & \sum_{i=1}^{n} B\left(0, T_{i}\right) e^{-\left(b_{0}+c_{0}\right) T_{i}}, \\
\widetilde{K}_{2}= & \sum_{i=1}^{n} E\left[D\left(T_{i}\right) L\left(T_{i-1}, T_{i-1}\right) 1_{\left\{\tau^{B} \wedge \tau^{C}>T_{i}\right\}}\right] \\
= & \sum_{i=1}^{n} L\left(T_{i-1}, T_{i-1}\right) B\left(0, T_{i}\right) e^{-\left(b_{0}+c_{0}\right) T_{i}}, \\
\widetilde{K}_{3}= & E\left[D\left(\tau^{C}+\delta\right) 1_{\left\{\tau^{C} \leq T\right\}} 1_{\left\{\tau^{B}>\tau^{C}+\delta\right\}}\right] \\
= & E\left[E\left[D\left(\tau^{C}+\delta\right) 1_{\left\{\tau^{C} \leq T\right\}} 1_{\left\{\tau^{B}>\tau^{C}+\delta\right\}} \mid \mathscr{F}_{T^{*}}^{r}\right]\right] \\
= & E\left[B\left(0, \tau^{C}+\theta\right) 1_{\left\{\tau^{C} \leq T\right\}} 1_{\left\{\tau^{B}>\tau^{C}+\theta\right\}}\right] \\
= & \int_{0}^{T} \int_{v+\theta}^{\infty} B(0, v+\theta) f(u, v) d u d v \\
= & \int_{0}^{T} B(0, v+\theta) \\
& \times \int_{v+\theta}^{\infty} c_{0} b_{0} b_{1}\left[t_{1}-t_{2}+\frac{1}{b_{1}}-\frac{1}{b_{0}}\right] e^{-\left(b_{0} t_{1}+c_{0} t_{2}\right)} d u d v \\
= & c_{0}\left(1+b_{1} \theta\right) e^{-b_{0} \theta} \int_{0}^{T} B(0, v+\theta) e^{-\left(b_{0}+c_{0}\right) v} d v .
\end{aligned}
$$

Substituting (36) into (18), we can have

$$
\begin{aligned}
s & =\frac{\sum_{i=1}^{n} C_{i} B\left(0, T_{i}\right) e^{-\left(b_{0}+c_{0}\right) T_{i}}}{\sum_{i=1}^{n} \delta M B\left(0, T_{i}\right) e^{-\left(b_{0}+c_{0}\right) T_{i}}} \\
& -\frac{\sum_{i=1}^{n} L\left(T_{i-1}, T_{i-1}\right) \delta M B\left(0, T_{i}\right) e^{-\left(b_{0}+c_{0}\right) T_{i}}}{\sum_{i=1}^{n} \delta M B\left(0, T_{i}\right) e^{-\left(b_{0}+c_{0}\right) T_{i}}} \\
& -\frac{c_{0}\left(1+b_{1} \theta\right) e^{-b_{0} \theta} \int_{0}^{T} B(0, v+\theta) e^{-\left(b_{0}+c_{0}\right) v} d v}{\sum_{i=1}^{n} \delta M B\left(0, T_{i}\right) e^{-\left(b_{0}+c_{0}\right) T_{i}}},
\end{aligned}
$$

which is formula (34). The proof is complete.

Remark 4. Combining formula (34) with formula (23), we can conclude that firm $C$ 's default contagion on firm $B$ has effect in the pricing of TRS (there is $b_{1}$ in formula (34)). However, firm $B$ 's default contagion on firm $C$ has no effect in the pricing of TRS (there is no $c_{1}$ in (34)). Therefore, in the complex contagion model, we can assume that the reference asset is the primary firm, and the protection seller is the secondary firm.

\section{TRS Valuation When Default Is Related to the Interest Rate}

Empirical studies show that in the vast majority of cases default is related to the interest rate or, in other words, credit risk is related to interest rate risk. In this section, we assume that their correlation is described by default intensities. For simplification, we assume that the length $\theta$ of the reference asset's settlement period is zero.

4.1. TRS Valuation under Two-Firm Looping Default Contagion Model. We consider that default intensities of firms $B$ and $C$ have the following form:

$$
\begin{aligned}
& \lambda_{t}^{B}=b_{0}+b r_{t}+b_{1} 1_{\left\{\tau^{C} \leq t\right\}}, \\
& \lambda_{t}^{C}=c_{0}+c r_{t}+c_{1} 1_{\left\{\tau^{B} \leq t\right\}},
\end{aligned}
$$

where $b_{0}, b_{0}, b$, and $c$ are nonnegative, satisfying $b_{0}+b_{1}>0$ and $c_{0}+c_{1}>0 . r_{t}=f(t, t)$ and $f(t, T)$ satisfies HJM model (9).

Using Wang and Ye's result [26], under the model (38), the joint conditional distribution of $\left(\tau^{B}, \tau^{C}\right)$ is given by

$$
\begin{aligned}
& P\left(\tau^{B}>t_{1}, \tau^{C}>t_{2} \mid \mathscr{F}_{T^{*}}^{r}\right) \\
&=\exp \left(-c_{0} t_{2}-c R_{0, t_{2}}\right) \times\left[\exp \left(-c_{1}\left(t_{2}-t_{1}\right)\right)-\exp \left(-\left(b_{0}\left(t_{2}-t_{1}\right)-b R_{t_{1}, t_{2}}\right)\right)\right. \\
& \quad+\exp \left(-b_{0} t_{2}-b R_{0, t_{2}}\right) \\
&\left.+c_{1} \int_{t_{1}}^{t_{2}} \exp \left(-b_{0}\left(u-t_{1}\right)-c_{1}\left(t_{2}-u\right)-b R_{t_{1}, u}\right) d u\right], \\
& P\left(\tau^{B}>\right.\left.t_{1}, \tau^{C}>t_{2} \mid \mathscr{F}_{T^{*}}^{r}\right) \\
&=\exp \left(-b_{0} t_{1}-b R_{0, t_{1}}\right) \\
& \times\left[\exp \left(-b_{1}\left(t_{1}-t_{2}\right)\right)-\exp \left(-\left(c_{2}\left(t_{1}-t_{2}\right)-c R_{t_{2}, t_{1}}\right)\right)\right. \\
& \quad+\exp \left(-c_{0} t_{1}-c R_{0, t_{1}}\right) \\
&\left.\quad+b_{1} \int_{t_{2}}^{t_{1}} \exp \left(-c_{0}\left(u-t_{2}\right)-b_{1}\left(t_{1}-u\right)-c R_{t_{2}, u}\right) d u\right], \\
& \quad \text { for } t_{2}<t_{1}<T .
\end{aligned}
$$


The joint conditional density function is

$$
\begin{aligned}
& f\left(t_{1}, t_{2} \mid \mathscr{F}_{T^{*}}^{r}\right)
\end{aligned}
$$

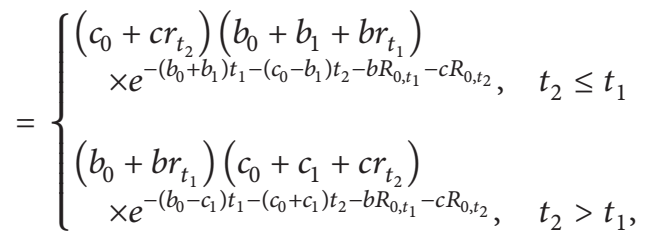

where $R_{0, u}=\int_{0}^{u} r_{v} d v$ is the cumulative interest rate process and $\mathscr{F}_{T^{*}}^{r}$ is the filter generated by $r_{t}$ up to $T^{*}$.

When default is related to interest rate, computing expectations in formula (18) becomes complicated. We give the following lemma first.

Lemma 5. The interest rate process is given by (9), $R_{t, s}=$ $\int_{t}^{s} r_{u} d u$, for $t<s$. Denote

$$
\begin{aligned}
G_{1}\left(m_{1} ; t_{0}, t_{1}\right) & =E\left[\exp \left(-m_{1} R_{t_{0}, t_{1}}\right)\right] \\
G_{2}\left(m_{1}, m_{2} ; t_{0}, t_{1}, t_{2}\right) & =E\left[\exp \left(-m_{1} R_{t_{0}, t_{1}}-m_{2} R_{t_{1}, t_{2}}\right)\right] ;
\end{aligned}
$$

then,

$$
\begin{gathered}
G_{1}\left(m_{1} ; t_{0}, t_{1}\right)=\exp \left(-m_{1} \int_{t_{0}}^{t_{1}} f\left(t_{0}, u\right) d u\right. \\
-m_{1} \int_{t_{0}}^{t_{1}} \int_{t_{0}}^{u} \alpha(s, u) d s d u \\
\left.+\frac{m_{1}^{2}}{2} \int_{t_{0}}^{t_{1}}\left(\int_{s}^{t_{1}} \sigma(s, u) d u\right)^{2} d s\right), \\
G_{2}\left(m_{1}, m_{2} ; t_{0}, t_{1}, t_{2}\right) \\
=\exp \left(-m_{1} \int_{t_{0}}^{t_{1}} f\left(t_{0}, u\right) d u\right. \\
\left.-m_{1} \int_{t_{0}}^{t_{1}} \int_{t_{0}}^{u} \alpha(s, u) d s d u\right)^{2} \\
\cdot \exp \left(-m_{2} \int_{t_{1}}^{t_{2}} f\left(t_{1}, u\right) d u\right. \\
\left.+m_{2} \int_{t_{1}}^{t_{2}} \int_{t_{1}}^{u} \alpha(s, u) d s d u\right)^{2} \int_{t_{1}}^{t_{1}}\left(\int_{s}^{t_{1}} \sigma(s, u) d u\right)^{2} d s \\
\left.\cdot \exp \left(\frac{m_{1}^{2}}{2} \int_{t_{0}}^{t_{2}} \sigma s\right) d u\right)^{2} d s
\end{gathered}
$$

Proof. Consider

$$
\begin{aligned}
R_{t_{0}, t_{1}}= & \int_{t_{0}}^{t_{1}} f\left(t_{0}, u\right) d u+\int_{t_{0}}^{t_{1}} \int_{t_{0}}^{u} \alpha(s, u) d s d u \\
& +\int_{t_{0}}^{t_{1}} \int_{t_{0}}^{u} \sigma(s, u) d W_{s} d u \\
= & \int_{t_{0}}^{t_{1}} f\left(t_{0}, u\right) d u+\int_{t_{0}}^{t_{1}} \int_{t_{0}}^{u} \alpha(s, u) d s d u \\
& +\int_{t_{0}}^{t_{1}} \int_{s}^{t_{1}} \sigma(s, u) d u d W_{s} .
\end{aligned}
$$

We can deduce

$$
\begin{aligned}
E\left[-m_{1} R_{t_{0}, t_{1}}\right] & =-m_{1} E\left[R_{t_{0}, t_{1}}\right] \\
& =-m_{1} \int_{t_{0}}^{t_{1}} f\left(t_{0}, u\right) d u-\int_{t_{0}}^{t_{1}} \int_{t_{0}}^{u} \alpha(s, u) d s d u \\
\operatorname{Var}\left[-m_{1} R_{t_{0}, t_{1}}\right] & =m_{1}^{2} \int_{t_{0}}^{t_{1}}\left(\int_{s}^{t_{1}} \sigma(s, u) d u\right)^{2} d s .
\end{aligned}
$$

By using the formula

$$
\begin{aligned}
E & {\left[\exp \left(-m_{1} R_{t_{0}, t_{1}}\right)\right] } \\
& =\exp \left(E\left[-m_{1} R_{t_{0}, t_{1}}\right]+\frac{1}{2} \operatorname{Var}\left[-m_{1} R_{t_{0}, t_{1}}\right]\right) .
\end{aligned}
$$

And substituting (45),(46) into (47), we can obtain (43).

Formula (43) can be rewritten as

$$
\begin{aligned}
G_{2}\left(m_{1}, m_{2} ; t_{0}, t_{1}, t_{2}\right) \\
\quad=E\left[\exp \left(-m_{1} R_{t_{0}, t_{1}}-m_{2} R_{t_{1}, t_{2}}\right)\right] \\
\quad=E\left[\exp \left(-m_{1} R_{t_{0}, t_{1}}\right) E_{t_{1}}\left[\exp \left(-m_{2} R_{t_{1}, t_{2}}\right)\right]\right] \\
\quad=E\left[\exp \left(-m_{1} R_{t_{0}, t_{1}}\right) L_{1}\left(m_{2} ; t_{1}, t_{2}\right)\right] .
\end{aligned}
$$

Similar to the computation of (42), (43) can be obtained, and we omit it here.

Since the length $\theta$ of settlement period for reference asset $C$ is 0 ; namely, if firm $B$ has no default, $B$ pays compensation for $C$ 's loss immediately once $C$ defaults, thus (18) becomes

$$
\begin{aligned}
s=(E & {\left[\sum_{i=1}^{n} D\left(T_{i}\right) C_{i} 1_{\left\{\tau^{B} \wedge \tau^{C}>T_{i}\right\}}\right] } \\
& \left.-E\left[\sum_{i=1}^{n} D\left(T_{i}\right) \delta M L\left(T_{i-1}, T_{i-1}\right) 1_{\left\{\tau^{B} \wedge \tau^{C}>T_{i}\right\}}\right]\right) \\
& \times\left(E\left[\sum_{i=1}^{n} D\left(T_{i}\right) \delta M 1_{\left\{\tau^{B} \wedge \tau^{C}>T_{i}\right\}}\right]\right)^{-1} \\
& -\frac{E\left[D\left(\tau^{C}\right) 1_{\left\{\tau^{C} \leq T\right\}} 1_{\left\{\tau^{B}>\tau^{C}\right\}}\right]}{E\left[\sum_{i=1}^{n} D\left(T_{i}\right) M 1_{\left\{\tau^{B} \wedge \tau^{C}>T_{i}\right\}}\right]} .
\end{aligned}
$$


Theorem 6. With default intensities (38) and HJM model (9), the price $s$ of TRS is given by

$$
\begin{aligned}
s= & \frac{\sum_{i=1}^{n} C_{i} \exp \left(-\left(b_{0}+c_{0}\right) T_{i}\right) \cdot G_{1}\left((1+b+c) ; 0, T_{i}\right)}{\sum_{i=1}^{n} \delta M \exp \left(-\left(b_{0}+c_{0}\right) T_{i}\right) \cdot G_{1}\left((1+b+c) ; 0, T_{i}\right)} \\
& -\left(\sum _ { i = 1 } ^ { n } M \left(G_{2}\left(1+b+c, b+c ; 0, T_{i-1}, T_{i}, t_{2}\right)\right.\right. \\
& \left.\left.-G_{1}\left(1+b+c ; 0, T_{i}\right)\right)\right) \\
& \times\left(\sum_{i=1}^{n} \delta M \exp \left(-\left(b_{0}+c_{0}\right) T_{i}\right) \cdot G_{1}\left((1+b+c) ; 0, T_{i}\right)\right)^{-1} \\
& \quad \frac{(c /(1+b+c))\left(1-e^{-(b+c) T} \cdot G_{1}(1+b+c ; 0, T)\right)}{\sum_{i=1}^{n} \delta M \exp \left(-\left(b_{0}+c_{0}\right) T_{i}\right) \cdot G_{1}\left((1+b+c) ; 0, T_{i}\right)} \\
- & \left(\left(c_{0}-\frac{c\left(b_{0}+c_{0}\right)}{1+b+c}\right) \times \int_{0}^{T} e^{-\left(b_{0}+c_{0}\right) v} \cdot G_{1}(1+b+c ; 0, v) d v\right) \\
& \times\left(\sum_{i=1}^{n} \delta M \exp \left(-\left(b_{0}+c_{0}\right) T_{i}\right) \cdot G_{1}\left((1+b+c) ; 0, T_{i}\right)\right)^{-1}
\end{aligned}
$$

Proof. Consider

$$
\begin{aligned}
s=(E & {\left[\sum_{i=1}^{n} D\left(T_{i}\right) C_{i} 1_{\left\{\tau^{B} \wedge \tau^{C}>T_{i}\right\}}\right] } \\
& \left.-E\left[\sum_{i=1}^{n} D\left(T_{i}\right) \delta M L\left(T_{i-1}, T_{i-1}\right) 1_{\left\{\tau^{B} \wedge \tau^{C}>T_{i}\right\}}\right]\right) \\
& \times\left(E\left[\sum_{i=1}^{n} D\left(T_{i}\right) \delta M 1_{\left\{\tau^{B} \wedge \tau^{C}>T_{i}\right\}}\right]\right)^{-1} \\
- & \frac{E\left[D\left(\tau^{C}\right) 1_{\left\{\tau^{C} \leq T\right\}} 1_{\left\{\tau^{B}>\tau^{C}\right\}}\right]}{E\left[\sum_{i=1}^{n} D\left(T_{i}\right) M 1_{\left\{\tau^{B} \wedge \tau^{C}>T_{i}\right\}}\right]}
\end{aligned}
$$

To compute (48), we need to only deduce the following three expectation values:

$$
\begin{aligned}
& \widehat{K}_{1}:=\sum_{i=1}^{n} E\left[D\left(T_{i}\right) 1_{\left\{\tau^{B} \wedge \tau^{C}>T_{i}\right\}}\right], \\
& \widehat{K}_{2}:=\sum_{i=1}^{n} E\left[D\left(T_{i}\right) L\left(T_{i-1}, T_{i-1}\right) 1_{\left\{\tau^{B} \wedge \tau^{C}>T_{i}\right\}}\right], \\
& \widehat{K}_{3}:=E\left[D\left(\tau^{C}\right) 1_{\left\{\tau^{C} \leq T\right\}} 1_{\left\{\tau^{B}>\tau^{C}\right\}}\right],
\end{aligned}
$$

where

$$
\begin{aligned}
\widehat{K}_{1} & =\sum_{i=1}^{n} E\left[D\left(T_{i}\right) 1_{\left\{\tau^{B} \wedge \tau^{C}>T_{i}\right\}}\right] \\
& =\sum_{i=1}^{n} E\left[E\left[D\left(T_{i}\right) 1_{\left\{\tau^{B} \wedge \tau^{C}>T_{i}\right\}} \mid \mathscr{F}_{T^{*}}^{r}\right]\right] \\
& =\sum_{i=1}^{n} E\left[e^{-R_{0, T_{i}}} \cdot E\left[1_{\left\{\tau^{B} \wedge \tau^{C}>T_{i}\right\}} \mid \mathscr{F}_{T^{*}}^{r}\right]\right] \\
& =\sum_{i=1}^{n} E\left[\exp \left(-\left(b_{0}+c_{0}\right) T_{i}\right) \cdot \exp \left(-(1+b+c) R_{0, T_{i}}\right)\right] \\
& =\sum_{i=1}^{n} \exp \left(-\left(b_{0}+c_{0}\right) T_{i}\right) \cdot G_{1}\left((1+b+c) ; 0, T_{i}\right), \\
\widehat{K}_{2} & =\sum_{i=1}^{n} E\left[D\left(T_{i}\right) L\left(T_{i-1}, T_{i-1}\right) 1_{\left\{\tau^{B} \wedge \tau^{C}>T_{i}\right\}}\right] \\
& =\sum_{i=1}^{n} E\left[E\left[D\left(T_{i}\right) L\left(T_{i-1}, T_{i-1}\right) 1_{\left\{\tau^{B} \wedge \tau^{C}>T_{i}\right\}} \mid \mathscr{F}_{T^{*}}^{r}\right]\right] .
\end{aligned}
$$

Substituting the expression (14) of Libor interest rate $L(t, T)$ into the formula above, we have

$$
\begin{gathered}
\widehat{K}_{2}=\sum_{i=1}^{n} E\left[E\left[e^{-R_{0, T_{i}}} \frac{1}{\delta}\left(e^{R_{T_{i-1}, T_{i-1}+\delta}}-1\right) 1_{\left\{\tau^{B} \wedge \tau^{C}>T_{i}\right\}} \mid \mathscr{F}_{T^{*}}^{r}\right]\right] \\
=\sum_{i=1}^{n} E\left[\frac{1}{\delta}\left(e^{-R_{0, T_{i-1}}}-e^{-R_{0, T_{i}}}\right) E\left[1_{\left\{\tau^{B} \wedge \tau^{C}>T_{i}\right\}} \mid \mathscr{F}_{T^{*}}^{r}\right]\right] \\
=\sum_{i=1}^{n} \frac{1}{\delta}\left(G_{2}\left(1+b+c, b+c ; 0, T_{i-1}, T_{i}\right)\right. \\
\left.\quad-G_{1}\left(1+b+c ; 0, T_{i}\right)\right),
\end{gathered}
$$

$$
\begin{aligned}
\widehat{K}_{3}=E & {\left[D\left(\tau^{C}\right) 1_{\left\{\tau^{C} \leq T\right\}} 1_{\left\{\tau^{B}>\tau^{C}\right\}}\right] } \\
= & E\left[E\left[D\left(\tau^{C}\right) 1_{\left\{\tau^{C} \leq T\right\}} 1_{\left\{\tau^{B}>\tau^{C}\right\}} \mid \mathscr{F}_{T^{*}}^{r}\right]\right] \\
=E & {\left[\int_{0}^{T} \int_{v}^{\infty} e^{-R_{0, v}} f\left(u, v \mid \mathscr{F}_{T^{*}}^{r}\right) d u d v\right] } \\
=E & {\left[\int_{0}^{T}\left(c_{0}+c r_{v}\right) e^{-\left(c_{0}-b_{1}\right) v-(1+c) R_{0, v}}\right.} \\
& \left.\quad \times \int_{v}^{\infty}\left(b_{0}+b_{1}+b r_{u}\right) e^{-\left(b_{0}+b_{1}\right) u-b R_{0, u}} d u d v\right]
\end{aligned}
$$




$$
\begin{aligned}
& =E\left[\left(c_{0}-\frac{c\left(b_{0}+c_{0}\right)}{1+b+c}\right)\right. \\
& \quad \times \int_{0}^{T} \exp \left(-\left(b_{0}+c_{0}\right) v\right) \exp \left(-(1+b+c) R_{0, v}\right) d v \\
& \quad+\frac{c}{1+b+c} \\
& \quad \times\left(\left(1-\exp \left(-\left(b_{0}+c_{0}\right) T-(1+b+c) R_{0, T}\right)\right)\right] \\
& =\frac{c}{1+b+c}\left(1-\exp \left(-\left(b_{0}+c_{0}\right) T\right)\right. \\
& \quad+\left(c_{0}-\frac{c\left(b_{0}+c_{0}\right)}{1+b+c}\right) \\
& \quad \times \int_{0}^{T} \exp \left(-\left(b_{0}+c_{0}\right) v\right) \cdot G_{1}(1+b+c ; 0, v) d v .
\end{aligned}
$$

Thus, substituting (52)-(54) into (48), we obtain

$$
\begin{aligned}
s= & \frac{\sum_{i=1}^{n} C_{i} \exp \left(-\left(b_{0}+c_{0}\right) T_{i}\right) \cdot G_{1}\left((1+b+c) ; 0, T_{i}\right)}{\sum_{i=1}^{n} \delta M \exp \left(-\left(b_{0}+c_{0}\right) T_{i}\right) \cdot G_{1}\left((1+b+c) ; 0, T_{i}\right)} \\
& -\left(\sum _ { i = 1 } ^ { n } M \left(G_{2}\left(1+b+c, b+c ; 0, T_{i-1}, T_{i}\right)\right.\right. \\
\left.\left.-G_{1}\left(1+b+c ; 0, T_{i}\right)\right)\right) & \\
& \times\left(\sum_{i=1}^{n} \delta M \exp \left(-\left(b_{0}+c_{0}\right) T_{i}\right) \cdot G_{1}\left((1+b+c) ; 0, T_{i}\right)\right)^{-1} \\
- & (c /(1+b+c))\left(1-e^{-(b+c) T} \cdot G_{1}(1+b+c ; 0, T)\right) \\
& \sum_{i=1}^{n} \delta M \exp \left(-\left(b_{0}+c_{0}\right) T_{i}\right) \cdot G_{1}\left((1+b+c) ; 0, T_{i}\right) \\
- & \left(\left(c_{0}-\frac{c\left(b_{0}+c_{0}\right)}{1+b+c}\right)\right. \\
& \left.\times\left(\sum_{i=1}^{n} \delta M \exp \left(-\left(b_{0}+c_{0}\right) T_{i}\right) \cdot G_{1}\left((1+b+c) ; 0, T_{i}\right)\right)^{-\left(b_{0}+c_{0}\right) v} \cdot G_{1}(1+b+c ; 0, v) d v\right)
\end{aligned}
$$

The proof is complete.

Remark 7. From the expression (49), the price $s$ of TRS is related to the default free interest rate risk (because there are $b$ and $c$ ). So the interest rate risk is not negligible in the pricing of credit derivatives.
4.2. TRS Valuation under Two-Firm Attenuation Contagion Model. In this subsection, besides that default is related to interest rate, we assume that default contagion has the hyperbolic attenuation effect. Default intensities of firms $B$ and $C$ are described as follows:

$$
\begin{aligned}
& \lambda_{t}^{B}=b_{0}+b r_{t}+\frac{b_{2}}{b_{1}\left(t-\tau^{C}\right)+1} 1_{\left\{\tau^{C} \leq t\right\}}, \\
& \lambda_{t}^{C}=c_{0}+c r_{t}+\frac{c_{2}}{c_{1}\left(t-\tau^{B}\right)+1} 1_{\left\{\tau^{B} \leq t\right\}},
\end{aligned}
$$

where $b_{0}, c_{0}, b, c, b_{1}$, and $c_{1}$ are nonnegative, satisfying $b_{0}+b_{2}>$ 0 and $c_{0}+c_{2}>0$. When $b_{1}=c_{1}=0$, model is simplified to the looping default model (38).

To obtain the analytic solution of $s$, we consider the following simplified model:

$$
\begin{aligned}
& \lambda_{t}^{B}=b_{0}+b r_{t}-\frac{b_{1}}{b_{1}\left(t-\tau^{C}\right)+1} 1_{\left\{\tau^{C} \leq t\right\}}, \\
& \lambda_{t}^{C}=c_{0}+c r_{t}-\frac{c_{1}}{c_{1}\left(t-\tau^{B}\right)+1} 1_{\left\{\tau^{B} \leq t\right\}} .
\end{aligned}
$$

By the result in [27], the joint conditional survival probability of $\left(\tau^{B}, \tau^{C}\right)$ under the model (57) is given by

$$
\begin{aligned}
& P(\left.\tau^{B}>t_{1}, \tau^{C}>t_{2} \mid \mathscr{F}_{T^{*}}^{r}\right) \\
&=\left(c_{1}\left(t_{2}-t_{1}\right)+1\right) e^{-\left(c_{0} t_{2}+c R_{0, t_{2}}\right)} \\
&+e^{-\left(b_{0}+c_{0}\right) t_{2}} \exp \left(-(b+c) R_{0, t_{2}}\right) \\
&-c_{1} e^{-c_{0} t_{2}} \int_{t_{1}}^{t_{2}} e^{-b_{0}\left(s-t_{1}\right)} \exp \left(-c R_{0, t_{2}}-b R_{t_{1}, s}\right) d s \\
&-e^{-b_{0}\left(t_{2}-t_{1}\right)-c_{0} t_{2}} \exp \left(-c R_{0, t_{2}}-b R_{t_{1}, t_{2}}\right) \\
& P\left(\tau^{B}>t_{1}, \tau^{C}>t_{2} \mid \mathscr{F}^{r}\right) \text { for } t_{1} \leq t_{2} \leq T, \\
&=\left(b_{1}\left(t_{1}-t_{2}\right)+1\right) e^{-\left(b_{0} t_{1}+b R_{0, t_{1}}\right)} \\
&+e^{-\left(b_{0}+c_{0}\right) t_{1}} \exp \left(-(b+c) R_{0, t_{1}}\right) \\
&-b_{1} e^{-b_{0} t_{1}} \int_{t_{2}}^{t_{1}} e^{-c_{0}\left(s-t_{2}\right)} \exp \left(-b R_{0, t_{1}}-c R_{t_{2}, s}\right) d s \\
&-e^{-c_{0}\left(t_{1}-t_{2}\right) b_{0} t_{1}} \exp \left(-b R_{0, t_{1}}-c R_{t_{2}, t_{1}}\right) \\
& \text { for } t_{2} \leq t_{1} \leq T,
\end{aligned}
$$


and the joint conditional density function is given by

$$
\begin{aligned}
& f\left(t_{1}, t_{2} \mid \mathscr{F}_{T^{*}}^{r}\right)
\end{aligned}
$$

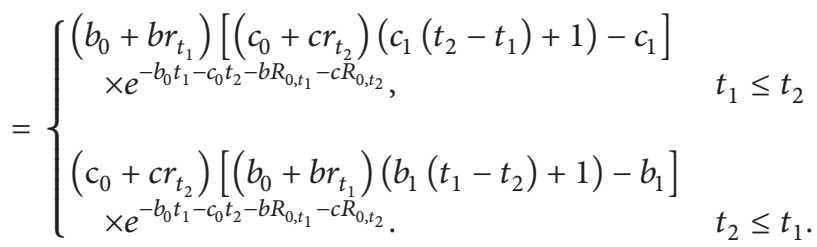

Under the model (57), the price formula of $s$ is given by

$$
\begin{aligned}
s=\left(E\left[\sum_{i=1}^{n} D\left(T_{i}\right) C_{i} 1_{\left\{\tau^{B} \wedge \tau^{C}>T_{i}\right\}}\right]\right. \\
\left.-E\left[\sum_{i=1}^{n} D\left(T_{i}\right) \delta M L\left(T_{i-1}, T_{i-1}\right) 1_{\left\{\tau^{B} \wedge \tau^{C}>T_{i}\right\}}\right]\right) \\
\\
\times\left(E\left[\sum_{i=1}^{n} D\left(T_{i}\right) \delta M 1_{\left\{\tau^{B} \wedge \tau^{C}>T_{i}\right\}}\right]\right)^{-1} \\
-\frac{E\left[D\left(\tau^{C}\right) 1_{\left\{\tau^{C} \leq T\right\}} 1_{\left\{\tau^{B}>\tau^{C}\right\}}\right]}{E\left[\sum_{i=1}^{n} D\left(T_{i}\right) \delta M 1_{\left\{\tau^{B} \wedge \tau^{C}>T_{i}\right\}}\right]}
\end{aligned}
$$

Combining with Lemma 5 , we can obtain the price $s$ of TRS, and the result is the following theorem.

Theorem 8. With default intensities (57) and HJM interest rate model (9), the price sof TRS is given by

$$
\begin{aligned}
s= & \frac{\sum_{i=1}^{n} C_{i} \exp \left(-\left(b_{0}+c_{0}\right) T_{i}\right) \cdot G_{1}\left((1+b+c) ; 0, T_{i}\right)}{\sum_{i=1}^{n} \delta M \exp \left(-\left(b_{0}+c_{0}\right) T_{i}\right) \cdot G_{1}\left((1+b+c) ; 0, T_{i}\right)} \\
& -\left(\sum _ { i = 1 } ^ { n } M \left(G_{2}\left(1+b+c, b+c ; 0, T_{i-1}, T_{i}\right)\right.\right. \\
\left.-G_{1}\left(1+b+c ; 0, T_{i}\right)\right) & \\
& \times\left(\sum_{i=1}^{n} \delta M \exp \left(-\left(b_{0}+c_{0}\right) T_{i}\right) \cdot G_{1}\left((1+b+c) ; 0, T_{i}\right)\right)^{-1}
\end{aligned}
$$

$$
\begin{aligned}
& -\frac{(c /(1+b+c))\left(1-e^{-(b+c) T} \cdot G_{1}(1+b+c ; 0, T)\right)}{\sum_{i=1}^{n} \delta M \exp \left(-\left(b_{0}+c_{0}\right) T_{i}\right) \cdot G_{1}\left((1+b+c) ; 0, T_{i}\right)} \\
& -\left(\left(c_{0}-\frac{c\left(b_{0}+c_{0}\right)}{1+b+c}\right)\right. \\
& \left.\quad \times \int_{0}^{T} e^{-\left(b_{0}+c_{0}\right) v} \cdot G_{1}(1+b+c ; 0, v) d v\right) \\
& \quad \times\left(\sum_{i=1}^{n} \delta M \exp \left(-\left(b_{0}+c_{0}\right) T_{i}\right) \cdot G_{1}\left((1+b+c) ; 0, T_{i}\right)\right)^{-1} .
\end{aligned}
$$

Proof. From (60), to obtain the analytic solution of $s$, we need to only compute three expectation values:

$$
\begin{aligned}
& \overline{K_{1}}:=\sum_{i=1}^{n} E\left[D\left(T_{i}\right) 1_{\left\{\tau^{B} \wedge \tau^{C}>T_{i}\right\}}\right], \\
& \overline{K_{2}}:=\sum_{i=1}^{n} E\left[D\left(T_{i}\right) L\left(T_{i-1}, T_{i-1}\right) 1_{\left\{\tau^{B} \wedge \tau^{C}>T_{i}\right\}}\right], \\
& \overline{K_{3}}:=E\left[D\left(\tau^{C}\right) 1_{\left\{\tau^{C} \leq T\right\}} 1_{\left\{\tau^{B}>\tau^{C}\right\}}\right],
\end{aligned}
$$

where

$$
\begin{aligned}
& \overline{K_{1}}=\sum_{i=1}^{n} E\left[D\left(T_{i}\right) 1_{\left\{\tau^{B} \wedge \tau^{C}>T_{i}\right\}}\right] \\
& =\sum_{i=1}^{n} E\left[E\left[D\left(T_{i}\right) 1_{\left\{\tau^{B} \wedge \tau^{C}>T_{i}\right\}} \mid \mathscr{F}_{T^{*}}^{r}\right]\right] \\
& =\sum_{i=1}^{n} E\left[e^{-R_{0, T_{i}}} \cdot E\left[1_{\left\{\tau^{B} \wedge \tau^{C}>T_{i}\right\}} \mid \mathscr{F}_{T^{*}}^{r}\right]\right] \\
& =\sum_{i=1}^{n} \exp \left(-\left(b_{0}+c_{0}\right) T_{i}\right) \cdot G_{1}\left((1+b+c) ; 0, T_{i}\right), \\
& \overline{K_{2}}=\sum_{i=1}^{n} E\left[D\left(T_{i}\right) L\left(T_{i-1}, T_{i-1}\right) 1_{\left\{\tau^{B} \wedge \tau^{C}>T_{i}\right\}}\right] \\
& =\sum_{i=1}^{n} E\left[E\left[\frac{1}{\delta} e^{-R_{0, T_{i}}}\left(e^{R_{T_{i-1}, T_{i-1}+\delta}}-1\right) 1_{\left\{\tau^{B} \wedge \tau^{C}>T_{i}\right\}} \mid \mathscr{F}_{T^{*}}^{r}\right]\right] \\
& =\sum_{i=1}^{n} \frac{1}{\delta}\left(G_{2}\left(1+b+c, b+c ; 0, T_{i-1}, T_{i}\right)\right. \\
& \left.-G_{1}\left(1+b+c ; 0, T_{i}\right)\right), \\
& \overline{K_{3}}=E\left[D\left(\tau^{C}\right) 1_{\left\{\tau^{C} \leq T\right\}} 1_{\left\{\tau^{B}>\tau^{C}\right\}}\right] \\
& =E\left[E\left[D\left(\tau^{C}\right) 1_{\left\{\tau^{C} \leq T\right\}} 1_{\left\{\tau^{B}>\tau^{C}\right\}} \mid \mathscr{F}_{T^{*}}^{r}\right]\right] \\
& =E\left[\left(c_{0}-\frac{c\left(b_{0}+c_{0}\right)}{1+b+c}\right)\right. \\
& \times \int_{0}^{T} \exp \left(-\left(b_{0}+c_{0}\right) v\right)
\end{aligned}
$$




$$
\begin{gathered}
\quad \times \exp \left(-(1+b+c) R_{0, v}\right) d v \\
+\frac{c}{1+b+c} \\
\times\left(1-\exp \left(-\left(b_{0}+c_{0}\right) T-(1+b+c) R_{0, T}\right)\right] \\
=\left(c_{0}-\frac{c\left(b_{0}+c_{0}\right)}{1+b+c}\right) \\
\times \int_{0}^{T} \exp \left(-\left(b_{0}+c_{0}\right) v\right) \cdot G_{1}(1+b+c ; 0, v) d v \\
+\frac{c}{1+b+c}\left(1-\exp \left(-\left(b_{0}+c_{0}\right) T\right)\right. \\
\left.\cdot G_{1}(1+b+c ; 0, T)\right) .
\end{gathered}
$$

Substituting expressions (63) of $\overline{K_{1}}, \overline{K_{2}}$, and $\overline{K_{3}}$ into (60), we can obtain (61). We complete the proof.

Remark 9. From formulas (49) and (61), we conclude that if the length $\theta$ of the reference asset's settlement period is 0 , the price $s$ of TRS is only related to interest rate risk and systematic risk.

\section{Conclusion}

In this paper, we mainly study the pricing of TRS under the framework of two-firm contagion models and HJM forward interest rate model. We obtain the analytic price expressions of TRS, respectively, based on whether the default is related to the interest rate. From these expressions, we claim that both default risk and the default-free interest rate risk have effects on the valuation of TRS. Moreover, the contagion effect between the reference asset and the protection seller is not ignorable. Therefore, the models in our paper have certain practical significance. We will further discuss other contagion models and interest rate models and compare them with the models in this paper by using Monte Carlo simulation.

\section{Conflict of Interests}

The authors declare that there is no conflict of interests regarding the publication of this paper.

\section{Acknowledgments}

The research was supported by the National Natural Science Foundation of China under Grants no. 11326168 and no. 11171215 and Yangcai Project (no. YC-XK-13106).

\section{References}

[1] R. C. Merton, "On the pricing of corporate debt: the risk structure of interest rates," The Journal of Finance, vol. 29, no. 2, pp. 449-470, 1974.
[2] F. Black and J. C. Cox, "Valuing corporate securities: some effects of bond indenture provisions," The Journal of Finance, vol. 31, no. 2, pp. 351-367, 2007.

[3] R. Geske, "The valuation of corporate liabilities as compound options," The Journal of Financial and Quantitative Analysis, vol. 12, no. 4, pp. 541-552, 1977.

[4] P. Artzner and F. Delbaen, "Default risk insurance and incomplete markets," Mathematical Finance, vol. 5, no. 3, pp. 187-195, 1995.

[5] D. Duffie, M. Schroder, and C. Skiadas, "Recursive valuation of defaultable securities and the timing of resolution of uncertainty," The Annals of Applied Probability, vol. 6, no. 4, pp. 10751090, 1996.

[6] R. A. Jarrow and S. M. Turnbull, "Pricing options on financial securities subject to default risk," The Journal of Finance, vol. 50, no. 1, pp. 53-86, 1995.

[7] D. B. Madan and H. Unal, "Pricing the risks of default," Review of Derivatives Research, vol. 2, no. 2-3, pp. 121-160, 1998.

[8] D. Duffie and D. Lando, "Term structures of credit spreads with incomplete accounting information," Econometrica, vol. 69, no. 3, pp. 633-664, 2001.

[9] R. A. Jarrow, D. Lando, and S. M. Turnbull, "A Markov model for the term structure of credit risk spreads," The Review of Financial Studies, vol. 10, no. 2, pp. 481-523, 1997.

[10] R. B. Litterman and T. Iben, "Corporate Bond Valuation and Term Structure of Credit Spreads, Financial Anal," The Journal of Portfolio Management, vol. 17, no. 3, pp. 52-64, 1991.

[11] D. Duffie and K. J. Singleton, "Modeling term structures of defaultable bonds," Review of Financial Studies, vol. 12, no. 4, pp. 687-720, 1999.

[12] D. Lando, "On cox processes and credit risky securities," Review of Derivatives Research, vol. 2, no. 2-3, pp. 99-120, 1998.

[13] P. Collin-Dufresne and B. Solnik, "On the term structure of default premia in the swap and LIBOR markets," The Journal of Finance, vol. 56, no. 3, pp. 1095-1115, 2001.

[14] G. R. Duffee, "Estimating the price of default risk," Review of Financial Studies, vol. 12, no. 1, pp. 197-226, 1999.

[15] R. A. Jarrow and F. Yu, "Counterparty risk and the pricing of defaultable securities," The Journal of Finance, vol. 56, no. 5, pp. 1765-1799, 2001.

[16] R. J. Elliott, M. Jeanblanc, and M. Yor, "On models of default risk," Mathematical Finance, vol. 10, no. 2, pp. 179-195, 2000.

[17] F. Jamshidian, Valuation of Credit Default Swaps and Swaptions, NIB Capital Bank, The Hague, The Netherlands, 2003.

[18] Z.X. Ye and R. X. Zhuang, "Pricing of total return swap," Chinese Journal of Applied Probability and Statistics, vol. 28, no. 1, pp. 7986, 2012.

[19] S. R. Das and R. K. Sundaram, "An integrated model for hybrid securities," Management Science, vol. 53, no. 9, pp. 1439-1451, 2007.

[20] J. M. Harrison and D. M. Kreps, "Martingales and arbitrage in multiperiod securities markets," Journal of Economic Theory, vol. 20, no. 3, pp. 381-408, 1979.

[21] J. M. Harrison and S. R. Pliska, "Martingales and stochastic integrals in the theory of continuous trading," Stochastic Processes and Their Applications, vol. 11, no. 3, pp. 215-260, 1981.

[22] D. Heath, R. Jarrow, and A. Morton, "Bond pricing and the term structure of interest rates: a new methodology for contingent claims valuation," Econometrica, vol. 60, no. 1, pp. 77-105, 1992.

[23] S. E. Shreve, Stochastic Calculus for Finance: Continuous-Time Models, Springer, New York, NY, USA, 2004. 
[24] S. Y. Leung and Y. K. Kwok, "Credit default swap valuation with counterparty risk," The Kyoto Economic Review, vol. 74, no. 1, pp. 25-45, 2005.

[25] Y.-F. Bai, X.-H. Hu, and Z.-X. Ye, "A model for dependent default with hyperbolic attenuation effect and valuation of credit default swap," Applied Mathematics and Mechanics, vol. 28, no. 12, pp. 1643-1649, 2007.

[26] A.-J. Wang and Z.-X. Ye, "The pricing of credit risky securities under stochastic interest rate model with default correlation," Applications of Mathematics, vol. 58, no. 6, pp. 703-727, 2013.

[27] R.-L. Hao and Z.-X. Ye, "The intensity model for pricing credit securities with jump diffusion and counterparty risk," Mathematical Problems in Engineering, vol. 2011, Article ID 412565, 16 pages, 2011. 


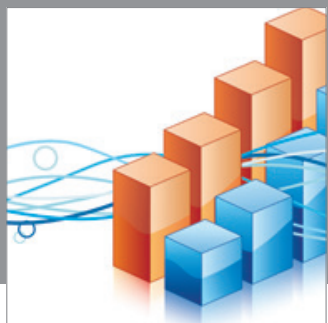

Advances in

Operations Research

mansans

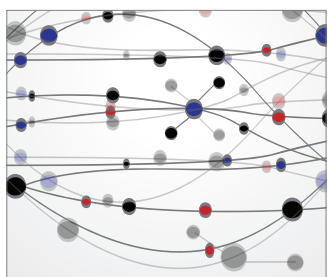

The Scientific World Journal
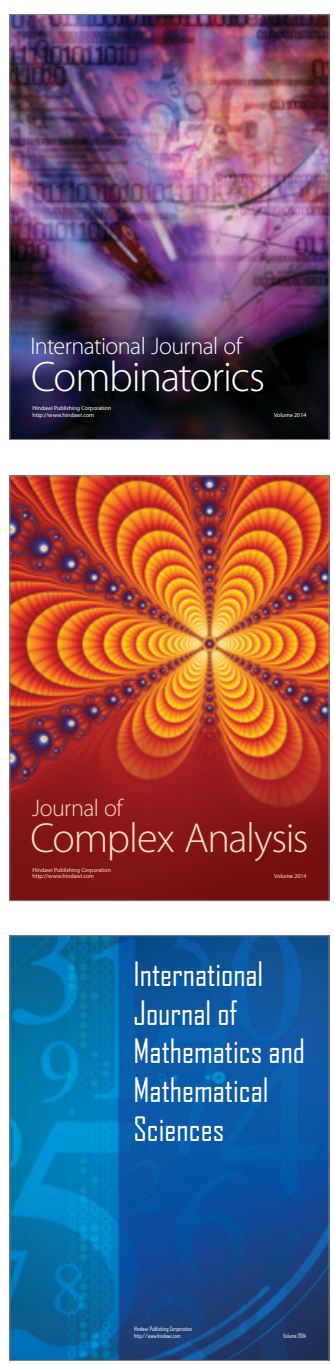
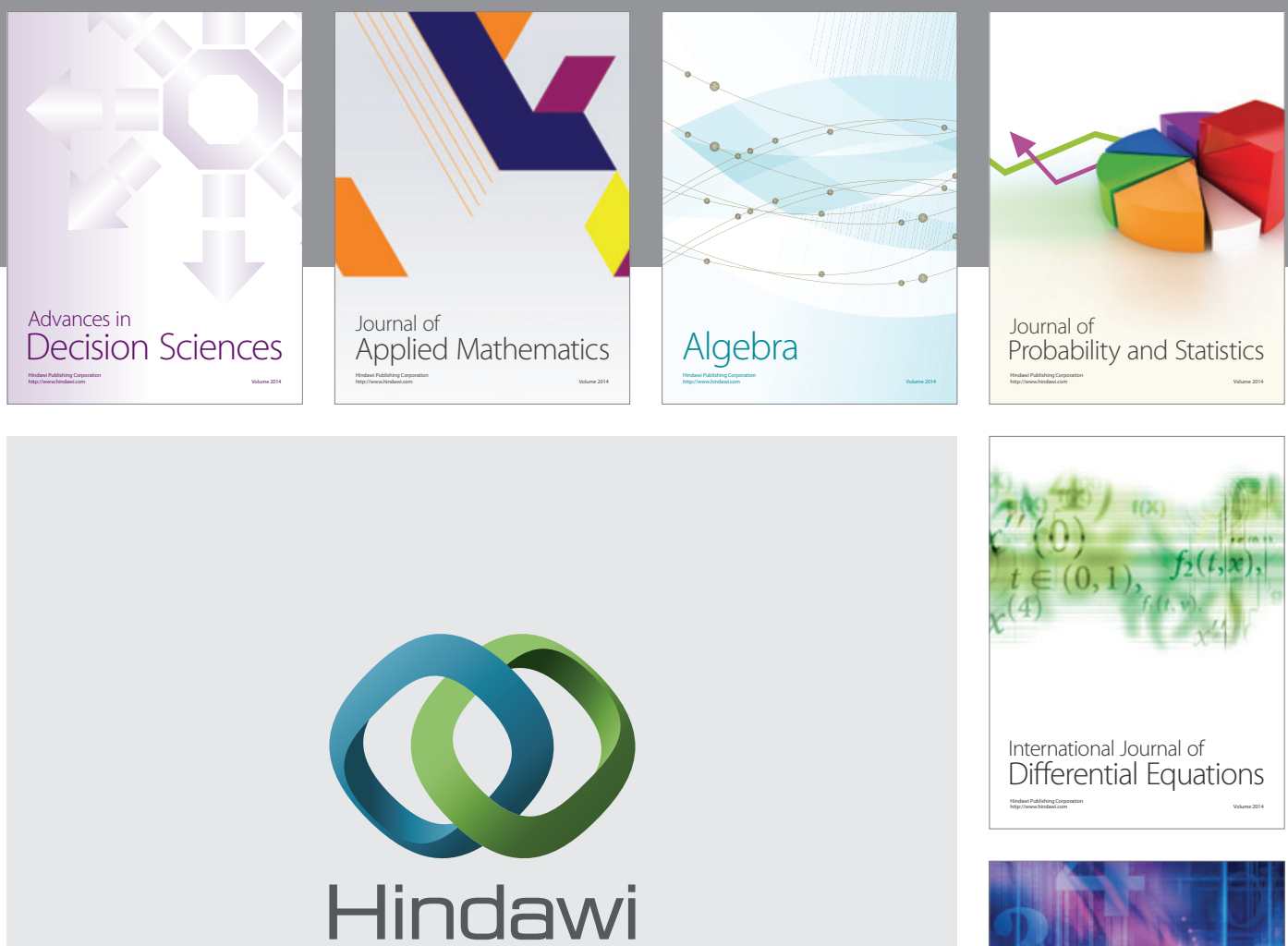

Submit your manuscripts at http://www.hindawi.com
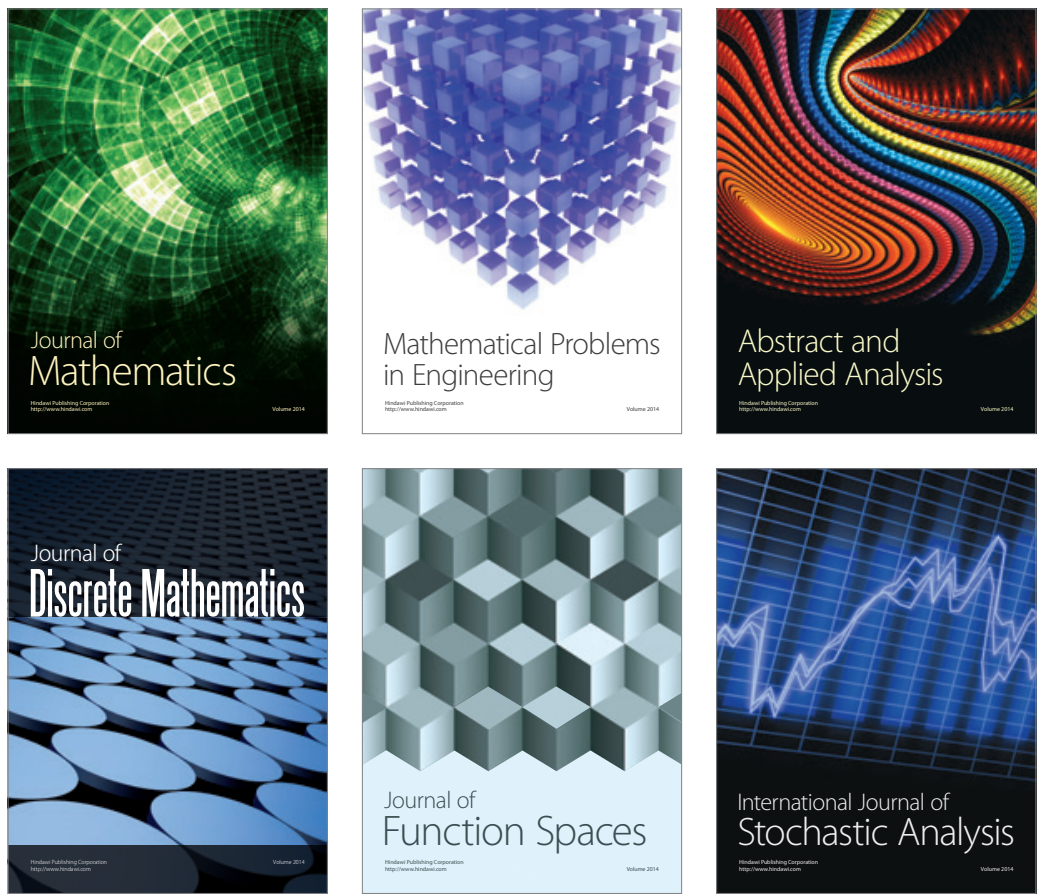

Journal of

Function Spaces

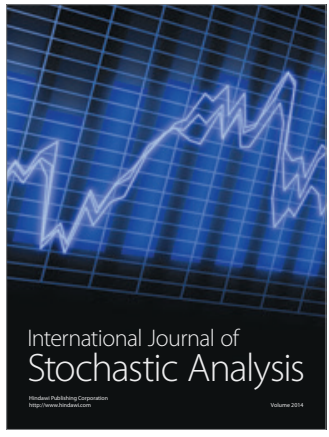

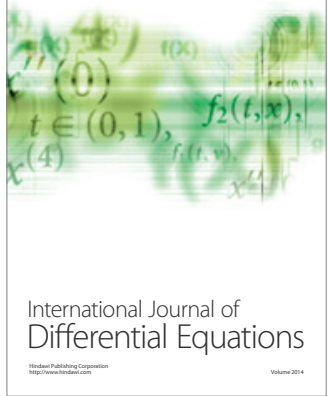
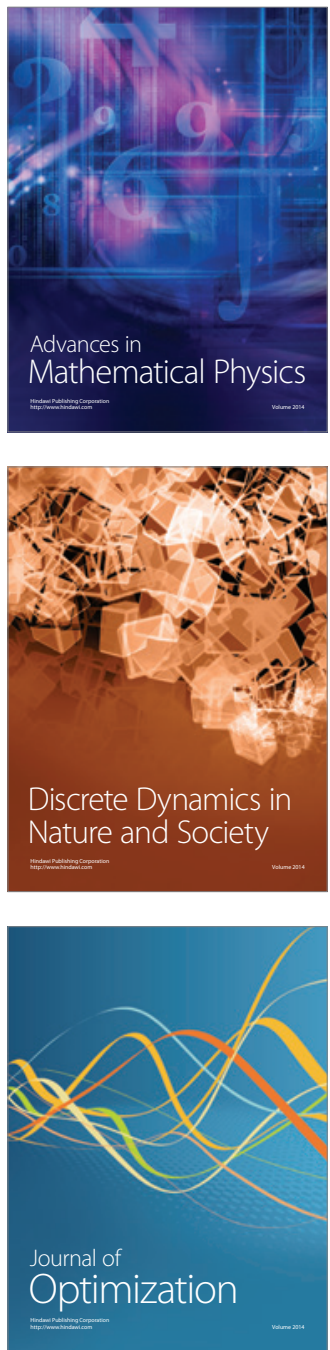